\title{
The course of chronic autoimmune thyroiditis in pregnant women during iodine and selenium therapy
}

\author{
Zsuzsanna Szántó1, Attila Csiszér ${ }^{2}$, Imre Zoltán Kun ${ }^{1}$, Zsuzsanna Croitorescu ${ }^{3}$
}

1. University of Medicine and Pharmacy, Tirgu Mures, Romania

2. Regional Center of Public Health Tirgu Mures, National Institute of Public Health, Tirgu Mures, Romania

3. Central Laboratory of Mures County Clinical Hospital, Tirgu Mures, Romania

\section{INTRODUCTION, OBJECTIVES}

Introduction. In iodine deficient regions maternal iodine supply is indispensable for the normal development of the fetus, but it might aggravate autoimmune thyroid diseases in the mother during pregnancy and postpartum. Selenium might be beneficial in these thyroid diseases by defending against the harmful effect of iodine on maternal thyroiditis.

Objective: to study the course of Hashimoto's thyroiditis in pregnant women, under combined therapy with L-thyroxin, selenium and iodine.

\section{MATERIAL AND METHODS}

In 13 pregnant women with chronic autoimmune thyroiditis we measured $\mathrm{TSH}$, free-T4, anti-thyroid peroxidase-antibody (TPO-Ab), anti-thyroglobulinantibody (Tg-Ab), serum selenium and urinary iodine concentration in the I-II trimester. Then I-thyroxin, selenium and iodine treatment was initiated, and thyroid function and the level of mentioned antibodies were followed in the IIIrd trimester and in the postpartum period.

\section{RESULTS}

Fig. 1. Distribution of high thyroid antibodies in our cases

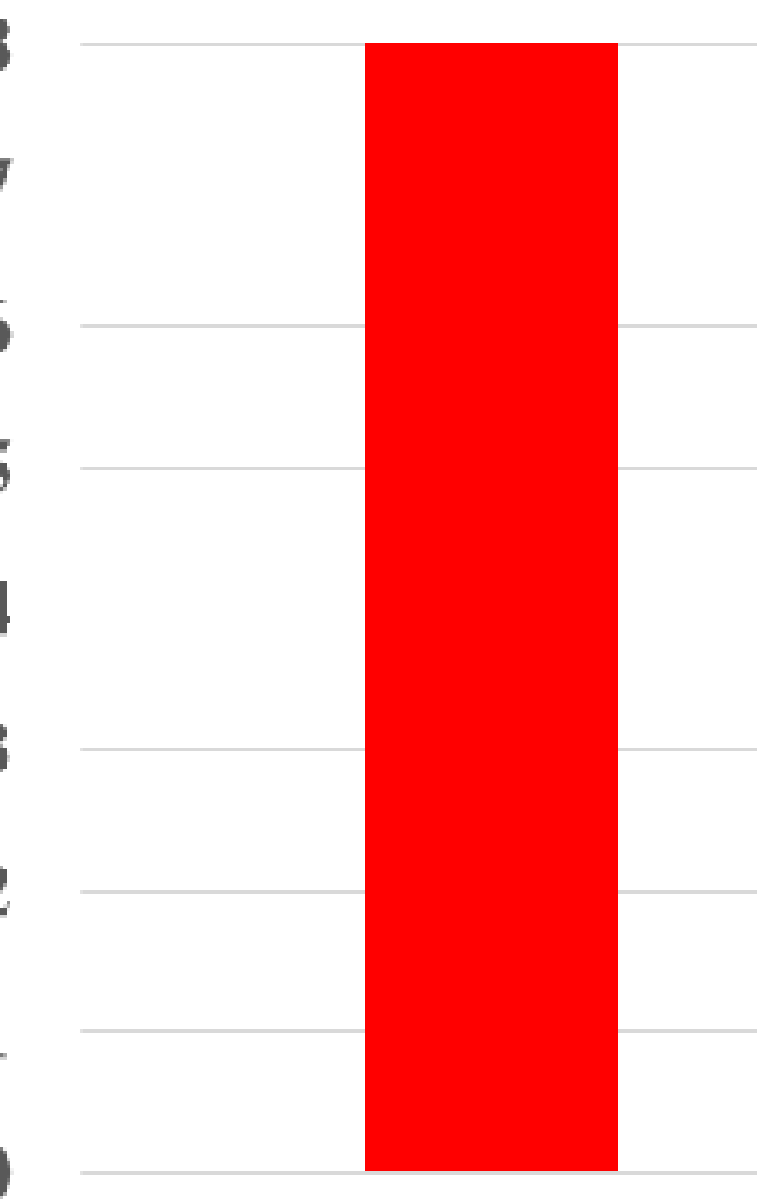

ATPO+aTg ATPO-aTg

The course of thyroid antibodies during pregnancy and postpartum period Fig 2. The variation of mean-TPO-Ab $(\mathrm{mIU} / \mathrm{L})$ during pregnancy

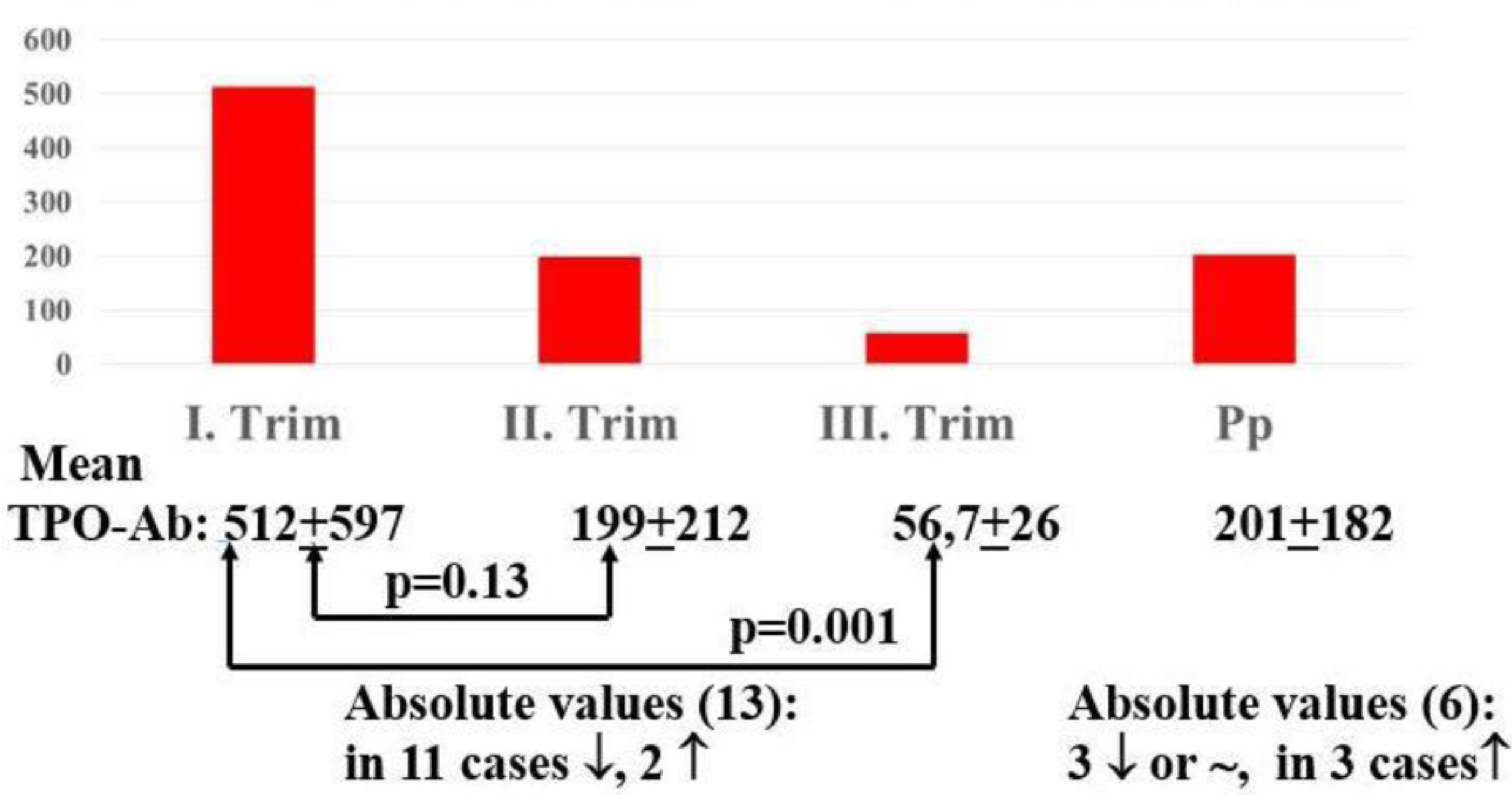

Fig. 3. The variation of Tg-Ab (mIU/L) during pregnancy
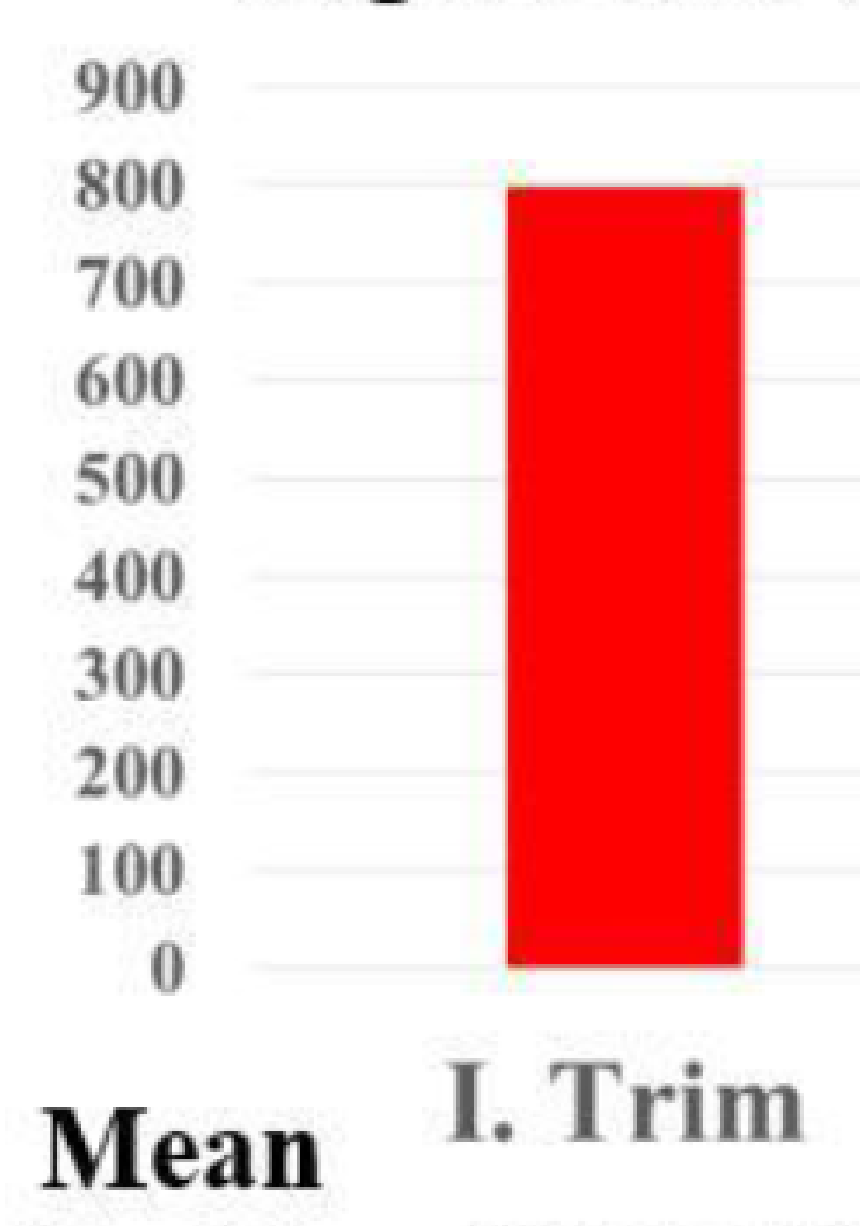

I. Trim

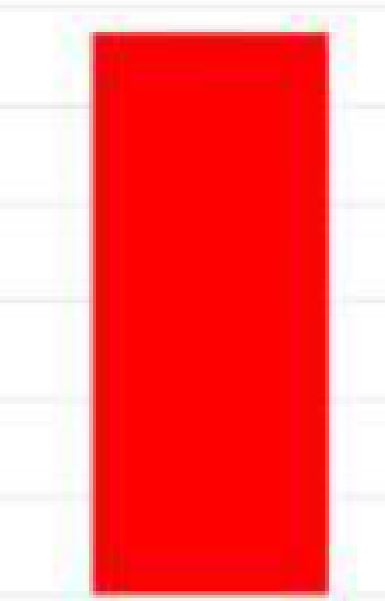

Tg-Ab:

II. Trim
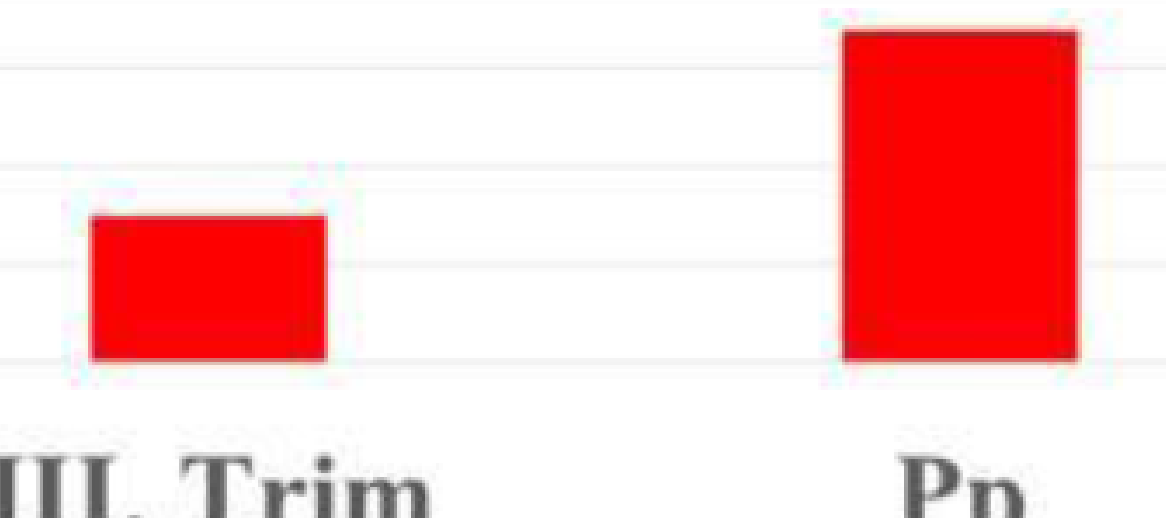

Trim

Pp

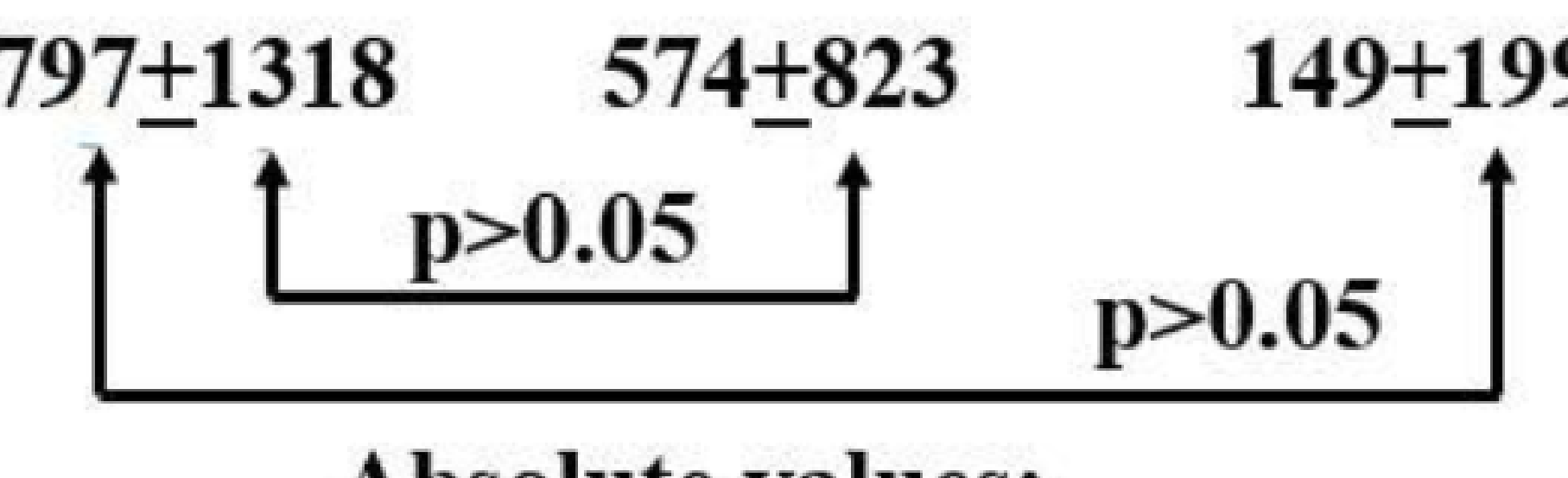

Absolute values: in 2 cases $\uparrow$

Absolute values (6): in 1 case $\uparrow$

Fig. 4. Selenium and iodine status in pregnant women

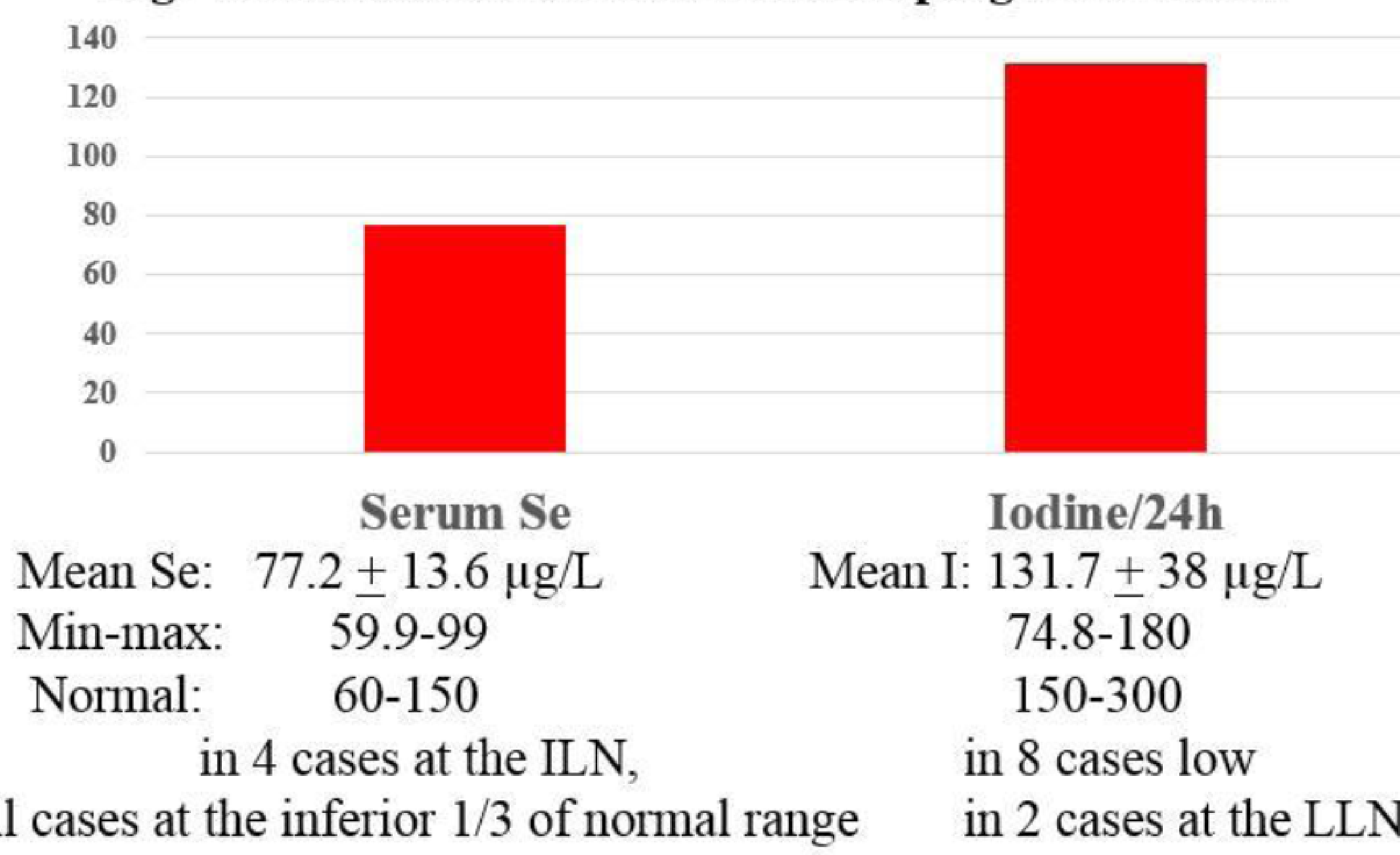

All women received 1-T4, and we combined to this iodine (100-200 microg/day) and selenium (50-200 microg/day). In all cases the level of thyroid antibodies decreased gradually during pregnancy, moreover in two cases it became normal, but after delivery all began to increase. Postpartum overt thyrotoxicosis developed in three cases $(25 \%)$, which improved spontaneously within 2 months (meanwhile iodine was reduced or stopped, and selenium continued).

\section{CONCLUSION}

Despite universal salt iodization implemented since 2002, iodine deficiency was detected in considerable part (8/13) of pregnant women with chronic autoimmune thyroiditis, requiring iodine supply. Combined therapy with L-thyroxine, selenium and iodine did not increase the level of thyroid autoantibodies during pregnancy, moreover it was decreased. Conversely, their level increased gradually in the postpartum period, when the maternal immunity return to pre-pregnant state, and in $25 \%$ of the cases overt thyrotoxicosis developed. 